\title{
Angiostrongylus chabaudi Biocca, 1957: a new parasite for domestic cats?
}

\author{
Antonio Varcasia ${ }^{1}$, Claudia Tamponi ${ }^{1}$, Emanuele Brianti $^{2}$, Piera Angela Cabras ${ }^{3}$, Roberta Boi ${ }^{3}$, Anna Paola Pipia ${ }^{1}$, \\ Alessio Giannelli ${ }^{4}$, Domenico Otranto ${ }^{4}$ and Antonio Scala ${ }^{1}$
}

\begin{abstract}
Background: Natural infection with a species of Angiostrongylus has been reported only once in wildcats from central Italy by Biocca in 1957. The causative species of this infection was identified as Angiostrongylus chabaudi. Following this report, this parasite had never been found in either wild or domestic cats.

Findings: The lungs and the pulmonary arteries of an adult female cat (Felis silvestris catus), road-killed in Sardinia, Italy, were macroscopically examined and dissected under a light microscope for the presence of parasites. A slender nematode was detected and its morphometrical features were consistent with those of A. chabaudi. Morphological data were supplemented by sequencing of the partial cytochrome oxidase c subunit 1 (cox1) gene, as well as the internal transcribed spacer 2 (ITS2) of the rDNA. Nucleotide sequences displayed $99 \%$ homology with the ITS2 sequence [GenBank KM216825.1] of a specimen of Angiostrongylus sp. recovered recently from the pulmonary artery of a wildcat in Germany and 91\% with cox1 sequence [GenBank GU138118.1] of Angiostrongylus vasorum.

Conclusion: The results of the present study indicate, for the first time, that A. chabaudi may also infect domestic cats, and thus should be considered in the diagnosis of metastrongyloid species infecting their cardio-pulmonary system.
\end{abstract}

Keywords: Angiostrongylus chabaudi, Metastrongyloidea, Angiostrongylidae, Cat, Cardio-pulmonary nematodes

\section{Findings}

Nematodes affecting the cardio-pulmonary system of pets have recently attracted the interest of researchers due to their increasing distribution in several European countries [1]. This is the case of Aelurostrongylus abstrusus and Eucoleus aerophilus (syn. Capillaria aerophila) in cats and of Angiostrongylus vasorum in dogs [2]. Recent reports of infections caused by other members of the superfamily Metastrongyloidea in domestic cats (e.g., Oslerus rostratus, Troglostrongylus brevior and Troglostrongylus subcrenatus) have further stimulated the interest of the scientific community on these little known species [3-7]. In addition, cats may act as permissive hosts of A. vasorum, although the first-stage larvae of this nematode are not shed in the faeces of experimentally infected animals [8,9]. A natural infection with a species of Angiostrongylus has been reported only once in wildcats from central Italy by Biocca in 1957, who described and named the causative species as

\footnotetext{
* Correspondence: ebrianti@unime.it

${ }^{2}$ Dipartimento di Scienze Veterinarie, Università degli Studi di Messina, 98168 Messina, Italy

Full list of author information is available at the end of the article
}

Angiostrongylus chabaudi [10]. Following this report, the species has never been found in either wild or domestic cats. Fifty-seven years after its description, we report the presence of $A$. chabaudi in a domestic cat from Sardinia together with the first molecular characterization of this little known angiostrongylid infecting cats.

The material originated from an adult female cat, road-killed in the municipality of Villacidro, province of Cagliari, Sardinia, Italy $\left(39^{\circ} 27^{\prime} 33^{\prime \prime} N\right.$, $\left.8^{\circ} 44^{\prime} 02 E^{\prime \prime}\right)$. Upon inspection of the pulmonary arteries, a slender nematode was retrieved. This parasite was washed in saline solution and subsequently mounted on a slide with glycerol, for microscopical observation. Light microscopy images and measurements were taken using a digital image processing system (Olympus BX41; Soft Imaging solution GMBH LG20, Munster, Germany). The parasite was identified to the species level using morphological keys $[10,11]$. A faecal sample was also collected from the rectum of the cat and processed by Baermann method for the detection of first-stage larvae of broncho-pulmonary nematodes [7]. The genetic identity of the cat (i.e., Felis silvestris silvestris, Felis silvestris lybica, Felis silvestris 


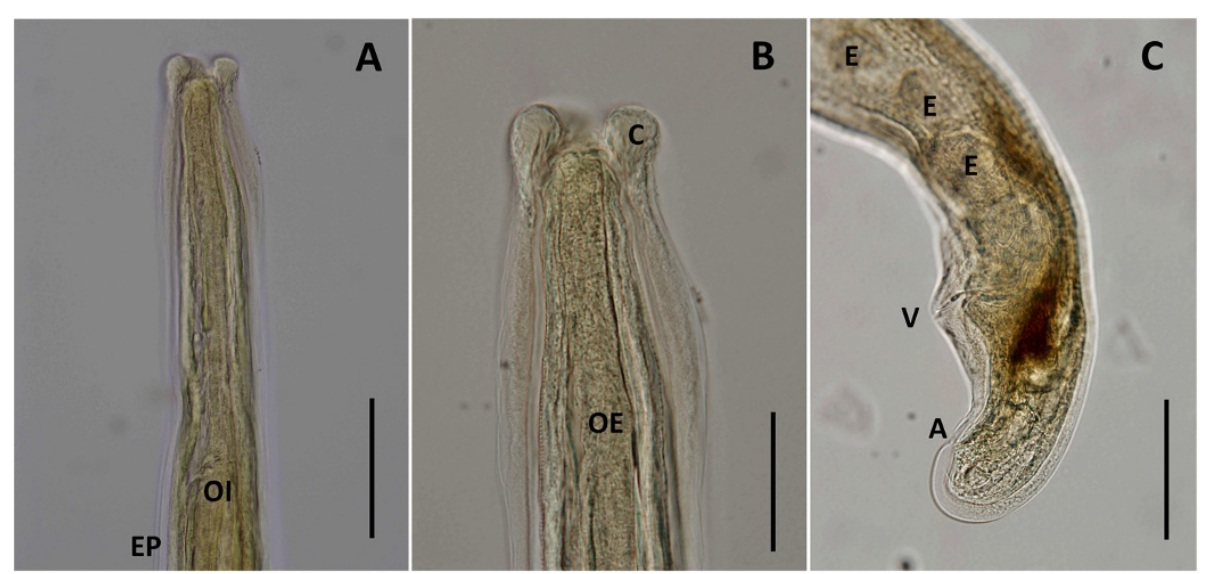

Figure 1 Angiostrongylus chabaudi. Light microscopy photomicrographs of female, lateral view. A. Cephalic region in lateral view; note the oesophageal-intestinal junction (OI) and level of the excretory pore (EP). B. Apical end, note dilatation of the cuticle resembling cephalic alae (C). C. Caudal end; note presence of eggs (E), vulvar aperture $(V)$ and anus (A). Scale bars: $\mathbf{A}=50 \mu \mathrm{m} ; \mathbf{B}-\mathbf{C}=100 \mu \mathrm{m}$.

catus or hybrid) was assessed molecularly on a muscle sample as described elsewhere [12].

Following parasite identification, DNA was extracted from the mid-body of the nematode using a commercial kit (High Pure PCR Template Preparation kit, Roche Diagnostics, Mannheim, Germany). Partial mithocondrial cytochrome $c$ oxidase subunit 1 ( $\operatorname{cox} 1$ ) gene and the internal transcribed spacer 2 (ITS2) of the rRNA gene were amplified, as previously described [13,14]. PCR amplicons were purified using an Exo SAP-IT kit (Amersham Biosciences) and sequenced through an external service (MWG Eurofins), using the same primers as for the PCR. Nucleotide sequences were compared with those available in GenBank $^{\circ}$ using the basic local alignment search tool (BLAST) analysis (National Centre for Biotechnology Information, ncbi.nlm.nih.gov). In order to investigate the relationships among metastrongyloids affecting wild and domestic carnivores, sequences of cox 1 and ITS2 were analysed with those available in GenBank ${ }^{\mathrm{Tx}}$. The evolutionary history was inferred for ITS2, using the maximum parsimony method using the Subtree-Pruning-Regrafting (SPR) algorithm with the software MEGA 6 [15]. The bootstrap consensus trees inferred from over 8,000 replicates were taken to represent the evolutionary history of the taxa analysed. The ITS2 sequence of Nematodirus battus was used as an outgroup.

The parasite specimen was a female with a slender and elongated body ( $16.9 \mathrm{~mm}$ long and $195.8 \mu \mathrm{m}$ wide). The anterior extremity was attenuated, whilst the posterior was rounded and curved forward (Figure 1). The cuticle was longitudinally striated and slightly dilated in the anterior end (Figure 1B). The buccal aperture, small and circular, was situated in the terminal position, surrounded by six perityls (Figure 2A). Two pairs of sensorial papillae (two ventral and two dorsal), each represented by two symmetrical conical protuberances, were noted posteriorly to the perityls (Figure 2A). The oesophagus was $276.6 \mu \mathrm{m}$ long and clavated. The excretory pore opened just behind the oesophageal-intestinal junction, at $361.5 \mu \mathrm{m}$ from the apical end. The vulva was sub-terminal and the anus was posterior to the vulvar aperture at $160.6 \mu \mathrm{m}$ and at $43.4 \mu \mathrm{m}$, respectively, from the caudal end (Figures $1 \mathrm{C}$ and $2 \mathrm{~B}$ ). Eggs (mean length $49.6 \pm 4.2 \mu \mathrm{m}$; mean width $33.4 \pm 0.9 \mu \mathrm{m}$ ) were seen inside the terminal tract of the uterus and in the ovijector (Figure 1C). The morphometrical features of the above parasite were consistent with those of $A$. chabaudi (Table 1) reported in the original description [10]. At the Baermann examination, only first-stage larvae of Troglostrongylus brevior were found and molecularly confirmed (data not shown). According to the molecular identification of the genotype, the cat was identified as pure domestic cat (F. silvestris catus).

The BLASTn of $\operatorname{cox} 1$ sequences herein obtained displayed a nucleotide homology of $91 \%$ with A. vasorum [GenBank GU138118.1], 89\% with Angiostrongylus cantonensis [GenBank GU138111.1] 88\% with A. abstrusus

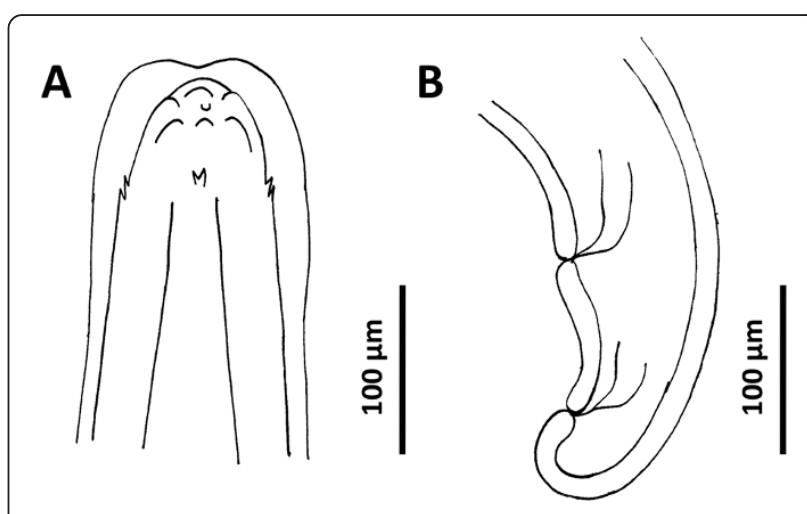

Figure 2 Angiostrongylus chabaudi, female. Schematic drawings. A. Anterior end, lateral view. B. Caudal end, lateral view. 
Table 1 Measurements for the known species of Angiostrongylus reported in carnivores

\begin{tabular}{|c|c|c|c|c|c|c|c|}
\hline Species & Present specimen & $\begin{array}{l}\text { Angiostrongylus } \\
\text { chabaudi [10] }\end{array}$ & Angiostrongylus sp. [16] & $\begin{array}{l}\text { Angiostrongylus } \\
\text { sp. [16] }\end{array}$ & $\begin{array}{l}\text { Angiostrongylus } \\
\text { vasorum [18] }\end{array}$ & $\begin{array}{l}\text { Angiostrongylus } \\
\text { daskalovi [18] }\end{array}$ & $\begin{array}{l}\text { Angiostrongylus } \\
\text { gubernaculatus [18] }\end{array}$ \\
\hline Host(s) & Domestic cat & Wildcat & Fox & Badger & $\begin{array}{l}\text { Dog, Coyote, } \\
\text { Fox, Jackal, Wolf }\end{array}$ & $\begin{array}{l}\text { Badger, Pine marten, } \\
\text { Beech marten }\end{array}$ & $\begin{array}{l}\text { American badger, } \\
\text { Striped skunk }\end{array}$ \\
\hline \multicolumn{8}{|l|}{ Males } \\
\hline Length (mm) & - & $14.6-16.3$ & $13.90 \pm 4.75(n=11)$ & $19.36 \pm 7.69(n=6)$ & $14.0-15.5$ & $13.36-21.31$ & $18-19.5$ \\
\hline Width (maximum) & - & $185-225$ & $221.40 \pm 12.13(n=11)$ & $243.20 \pm 19.63(n=6)$ & $170-235$ & $254-306$ & $300-335$ \\
\hline Oesophagus length & - & $300-345$ & $255.33 \pm 13.60(n=8)$ & $333.30 \pm 18.24(n=6)$ & $220-275$ & $336-366$ & $300-325$ \\
\hline $\begin{array}{l}\text { Distance from excretory pore to } \\
\text { cephalic end }\end{array}$ & - & $335-405$ & $356.50 \pm 34.68(n=4)$ & $409.5 \pm 25.50(n=2)$ & $310-350$ & $386-463$ & - \\
\hline Spicules length & - & $510-555$ & $411.06 \pm 17.71(n=10)$ & $345.57 \pm 23.95(n=8)$ & $400-500$ & $336-409$ & $520-560$ \\
\hline \multicolumn{8}{|l|}{ Females } \\
\hline Length (mm) & 16.9 & $19.1-24.1$ & $18.97 \pm 10.21(n=22)$ & $24.76 \pm 13.66(n=16)$ & $15-20.5$ & $14.39-31.12$ & $22-24$ \\
\hline Width (maximum) & 192.2 & $245-298$ & $304.14 \pm 14.84(n=18)$ & $344.52 \pm 17.95(n=17)$ & $220-306$ & $340-511$ & 350 \\
\hline Oesophagus length & 276.6 & $345-380$ & $279.88 \pm 20.27(n=14)$ & $368.45 \pm 17.00(n=16)$ & $240-280$ & $356-556$ & $335-350$ \\
\hline $\begin{array}{l}\text { Distance from excretory pore to } \\
\text { cephalic end }\end{array}$ & 361.5 & $395-470$ & $391.86 \pm 21.44(n=7)$ & $447.40 \pm 101.72(n=5)$ & $350-370$ & $379-636$ & - \\
\hline Distance from vulva to anus & 117.2 & - & $233.63 \pm 32.41(n=17)$ & $295.15 \pm 46.25(n=16)$ & $150-220$ & - & - \\
\hline Distance from anus to caudal end & 43.4 & $62-75$ & $69.70 \pm 2.87(n=17)$ & $78.82 \pm 3.45(n=16)$ & $67-100$ & $76-115$ & $75-95$ \\
\hline Distance from vulva to caudal end & 160.6 & $170-210$ & $275.66 \pm 30.75(n=18)$ & $366.25 \pm 44.17(n=16)$ & $220-315$ & $269-412$ & - \\
\hline
\end{tabular}

All measurements are given in micrometers $(\mu \mathrm{m})$ unless otherwise specified. 
[GenBank KF316481.1] and of 87\% with Angiostrongylus costaricensis [GenBank GU138117.1]. Interestingly, the ITS2 sequence displayed 99\% nucleotide identity with ITS2 of Angiostrongylus sp. [GenBank KM216825.1], recovered recently from the pulmonary artery of a wildcat in Germany and identity of $87 \%$ with A. vasorum [GenBank GU045375.1]. The ITS2 sequence of $A$. chabaudi displayed lower levels of homology of $79 \%, 85 \%$ and $79 \%$, respectively, with $A$. dujardini [GenBank GQ181113.1], A. costaricensis [GenBank LK942974.1] and A. cantonensis [GenBank LK949842.1].

Pairwise distances, calculated using the common region of cox 1 (454 bp) varied from 0.08 with $A$. vasorum [GenBank EU493161.1] to 0.13 with both $A$. cantonensis [GenBank GU138111.1] and A. abstrusus [GenBank KF316481.1] and of 0.17 with A. costaricensis [GenBank GU138116.1], whereas those based on ITS2 were identical with a sequence for Angiostrongylus sp. [GenBank KM216825.1] and diverged by 0.08 from that for $A$. vasorum [GenBank GU045375.1]. Analysis of the cox1 sequences clustered $A$. chabaudi in the clade including A. vasorum but this was not optimal due to the small number of sequences available (data not shown). Conversely, ITS2 phylogeny clearly indicated that $A$. chabaudi clustered within the clade of all other Angiostrongylus species available in public databases (Figure 3 ).
Fifty-seven years after its original description [10], $A$. chabaudi is reported here for the first time in a domestic cat from Sardinia. In addition, the present study represents the first report, ever, of an angiostrongylid of the genus Angiostrongylus naturally infecting domestic cat. The recovery of this nematode is of importance as it suggests that cats may also be infected by metastrongyloids localizing in the cardio-vascular system. Morphological features and size of the parasite found in the present survey are similar to those reported in the original description of $A$. chabaudi [10] (Table 1). Although eggs were observed in the uterus of the specimen herein studied, the absence of specific first-stage larvae at the Baermann test indicates that the parasite was an immature or unfertilized female, thereby explaining its smaller size compared to that previously recorded [10]. The morphometry of the parasite here described differs from that of A. vasorum in dogs and wildlife hosts (Table 1) [16], in that four sensorial papillae are present in the anterior region posteriorly to perityls, and the distance between the vulvar aperture and the caudal extremity (i.e., $160.6 \mu \mathrm{m}$ ) is significantly shorter than in other species of Angiostrongylus but similar to that of A. chabaudi (170-210 $\mu \mathrm{m})$ (Table 1). These morphological differences were also clearly supported by the nucleotide homology with sequences from other species (i.e., up to $91 \%$ for $\operatorname{cox} 1$

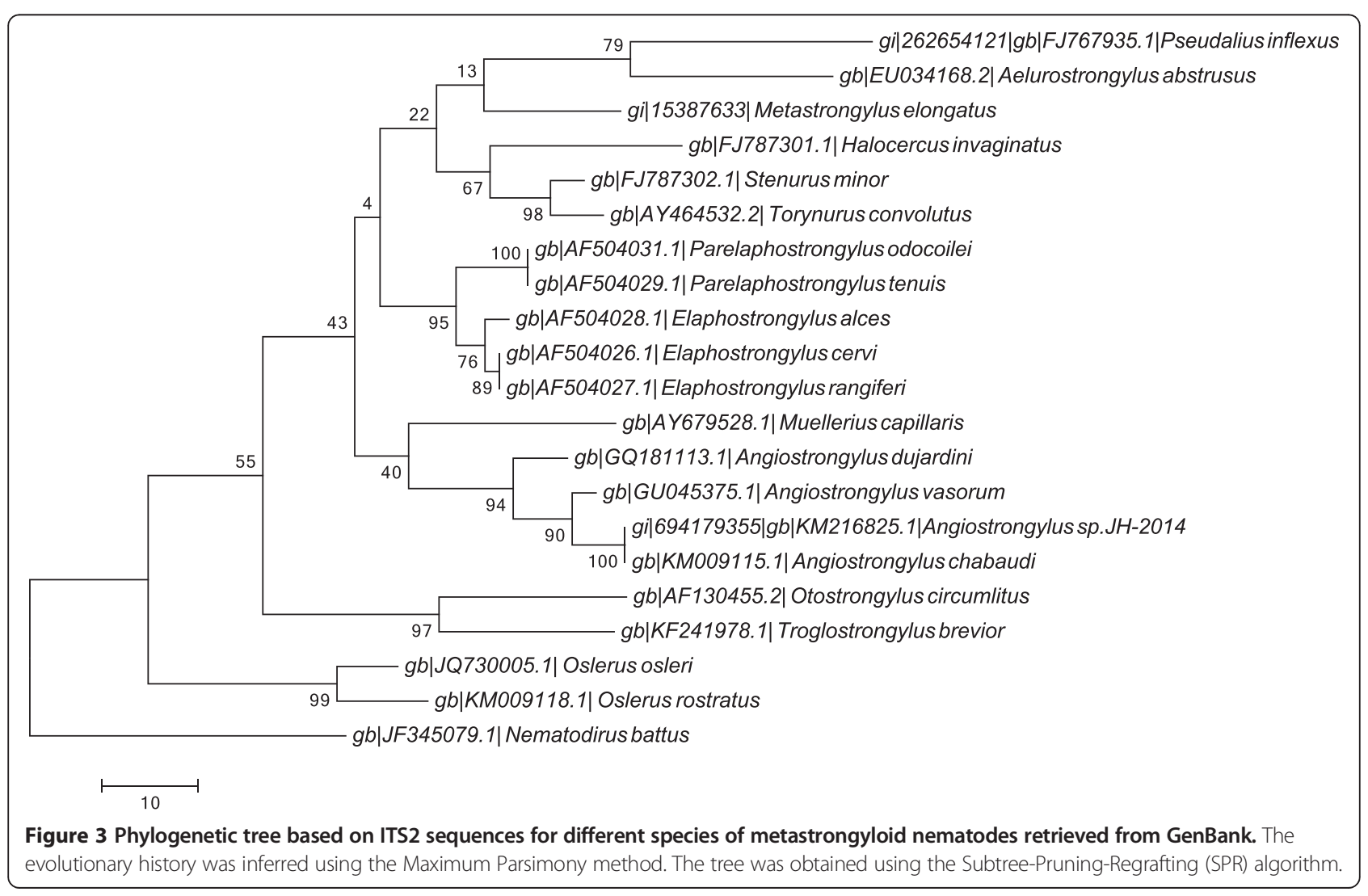


sequences with that of $A$. vasorum). The phylogenetic analyses, based on ITS2, supported their morphological identification, in that sequences derived from this nematode were clustered within those belonging to the genus Angiostrongylus.

In the original description, $A$. chabaudi was considered typical for wildcats, having been found in $85 \%$ of a sample from central Italy, but not in stray cats and dogs or in other wildlife species (i.e., Meles meles and Vulpes vulpes) from the same area [10].

Here A. chabaudi is reported for the first time in a domestic cat. No data are available on the presence of this nematode in wildcats from Sardinia, which belong to another subspecies (F. silvestris lybica) than that found in continental Italy [17]. Therefore, at least for Sardinia, it is not yet possible to infer that wildcats may be regarded as wild reservoirs of $A$. chabaudi. On the other hand, according to Biocca's study [10], wildcats may be highly infected by $A$. chabaudi. In addition, the ITS2 sequence of A. chabaudi herein characterized displayed a very high nucleotide homology (99\%) with that of Angiostrongylus sp. collected from a wildcat in Germany. Also, both nematodes above were collected from the pulmonary artery, which is the same site of collection of $A$. chabaudi as described by Biocca [10].

The data presented here suggest that a careful inspection of the vascular system of lungs should be undertaken during necropsies of domestic and wild cats. Also, firststage larvae of $A$. chabaudi have not been described so far [10] and thus their morphological delineation from those of other metastrongyloids affecting felids warrants investigation. Additional studies should elucidate the life history of A. chabaudi, determine its distribution and impact of this little known parasite on the health of wild and domestic cats.

\section{Competing interests}

The authors declare that they have no competing interests.

\section{Authors' contributions}

$A V, E B, C T$ conceived the study, described parasites and drafted the manuscript. PAC and RB collected parasites, CT, EB, DO, performed the morphological identification and revised the drafts of the manuscript. APP and AV carried out the molecular genetic studies, sequence alignment and phylogenetic analyses. AG and AS revised the drafts of manuscript. All authors read and approved the final version of the manuscript.

\section{Acknowledgements}

The research was funded by the Regional Government of Sardinia, prot. CRP2 134 (L.R. 7, 2007). The authors thank Miss Claudia Serra (University of Sassari, Italy) for the parasite drawings, Dr. Stefania Latrofa and Dr. Bronwyn Campbell (University of Bari, Italy) for revising the English and the phylogenetical analysis. Authors thanks Dr. Ettore Randi and Dr. Federica Mattucci, Istituto Superiore per la Protezione e la Ricerca Ambientale (ISPRA), Bologna, Italy for the genetic analyses performed to assess cat genotype.

\section{Author details}

'Laboratorio di Parassitologia, Ospedale Didattico Veterinario, Dipartimento di Medicina Veterinaria, Università degli Studi di Sassari, 07100 Sassari, Italy. ${ }^{2}$ Dipartimento di Scienze Veterinarie, Università degli Studi di Messina, 98168
Messina, Italy. ${ }^{3}$ Istituto Zooprofilattico Sperimentale della Sardegna, 08048 Tortoli, Italy. ${ }^{4}$ Dipartimento di Medicina Veterinaria, Università degli Studi di Bari, 70010 Valenzano, Bari, Italy.

Received: 29 October 2014 Accepted: 2 December 2014 Published online: 17 December 2014

\section{References}

1. Conboy G: Helminth parasites of the canine and feline respiratory tract. Vet Clin North Am Small Anim Pract 2009, 39:1109-1126.

2. Traversa D, Di Cesare A, Conboy G: Canine and feline cardiopulmonary parasitic nematodes in Europe: emerging and underestimated. Parasit Vectors 2010, 3:62.

3. Millán J, Casanova JC: High prevalence of helminth parasites in feral cats in Majorca Island (Spain). Parasitol Res 2009, 106:183-188.

4. Brianti E, Gaglio G, Giannetto S, Annoscia G, Latrofa MS, Dantas-Torres F, Traversa D, Otranto D: Troglostrongylus brevior and Troglostrongylus subcrenatus (Strongylida: Crenosomatidae) as agents of broncho-pulmonary infestation in domestic cats. Parasit Vectors 2012, 5:178.

5. Brianti E, Gaglio G, Napoli E, Falsone L, Giannelli A, Annoscia G, Varcasia A, Giannetto S, Mazzullo G, Otranto D: Feline lungworm Oslerus rostratus (Strongylida: Filaridae) in Italy: first case report and histopathological findings. Parasitol Res 2014, 113:3853-3857.

6. Otranto D, Brianti E, Dantas-Torres F: Troglostrongylus brevior and a nonexistent 'dilemma'. Trends Parasitol 2013, 29:517-518.

7. Tamponi C, Varcasia A, Brianti E, Pipia AP, Frau V, Pinna Parpaglia ML, Sanna G, Garippa G, Otranto D, Scala A: New insights on metastrongyloid lungworms infecting cats of Sardinia, Italy. Vet Parasitol 2014, 203:222-226.

8. Guilhon J, Cens B: Essais de transmission d'Angiostrongylus vasorum (Baillet, 1866) du chat. Comp Rend Acad Sci 1970, 271:936-939.

9. Dias SRC, Oliveira EL, Viana MH, Lima WS: Permissivity of the domestic cat (Felis catus) to infection by Angiostrongylus vasorum (Nematoda: Protostrongylidae). Revue Méd Vet 2008, 159:87-90.

10. Biocca E: Angiostrongylus chabaudi n. sp. parassita del cuore e dei vasi polmonari del gatto selvatico (Felis silvestris). R Accad Naz Lincei 1957, 22:526-532.

11. Costa JO, De Araújo Costa HM, Guimarães MP: Redescription of Angiostrongylus vasorum (Baillet, 1866) and systematic revision of species assigned to the genera Angiostrongylus Kamensky, 1905 and Angiocaulus Schulz, 1951. Revue Méd Vét 2003, 154:9-16.

12. Mattucci F, Oliveira R, Bizzarri L, Vercillo F, Anile S, Ragni B, Lapini L, Sforzi A, Alves PC, Lyons LA, Randi E: Genetic structure of wildcat (Felis silvestris) populations in Italy. Ecol Evol 2013, 3:2443-2458.

13. Gasser RB, Chilton NB, Hoste H, Beveridge I: Rapid sequencing of rDNA from single worms and eggs of parasitic helminths. Nucleic Acids Res 1993, 21:2525-2526.

14. Caldeira RL, Carvalho OS, Mendonça CL, Graeff-Teixeira C, Silva MC, Ben R, Maurer R, Lima WS, Lenzi HL: Molecular differentiation of Angiostrongylus costaricensis, $A$. cantonensis, and $A$. vasorum by polymerase chain reaction-restriction fragment length polymorphism. Mem Inst Oswaldo Cruz 2003, 98:1039-1043.

15. Tamura K, Stecher G, Peterson D, Filipski A, Kumar S: MEGA6: molecular evolutionary genetics analysis version 6.0. Mol Biol Evol 2013, 30:2725-2729.

16. Gerrikagoitia X, Barral M, Juste RA: Angiostrongylus species in wild carnivores in the Iberian Peninsula. Vet Parasitol 2010, 174:175-180.

17. Janchev J, Genov T: Angiostrongylus daskalovi sp. n. Nematoda: Filaroididae from Mustelidae in Bulgaria. Helminthologia 1988, 252:81-88.

18. Randi E, Pierpaoli M, Beaumont M, Ragni B, Sforzi A: Genetic identification of wild and domestic cats (Felis silvestris) and their hybrids using Bayesian clustering methods. Mol Biol Evol 2001, 18:1679-1693. 